\title{
SUPERHARMONIC FUNCTIONS AND BOUNDED POINT EVALUATIONS
}

\section{EDWIN WOLF}

\author{
Department of Mathematics \\ University of Lowe 11 \\ Lowell, Massachusetts 01854 \\ (Received October 18, 1988)
}

ABSTRACT. Let $E$ be a compact subset of the complex plane $\mathbb{~}$. We denote by $R_{0}(E)$ the algebra consisting of the (restrictions to $E$ of) rational functions with poles of E. Let $m$ denote the 2-dimensional Lebesgue measure. Let $R^{2}(E)$ be the closure of $R_{0}(E)$ in $L^{2}(E, d m)$.

In this paper we consider points $x \in E$ such that "evaluation at $x$ " extends from $R_{0}(E)$ to a continuous linear functional on $R^{2}(E)$. These points are bounded point evaluations on $\mathrm{R}^{2}(E)$. Hedberg, Fernström and Polking used capacity to identify bounded point evaluations. We use their results to show that the existence of a bounded point evaluation $x \in E$ is equivalent to the existence of a superharmonic function $u(y)$ that grows sufficiently fast as $y$ approaches $x$ through the complement of E.

KEY WORDS AND PHRASES. Rational functions, compact set, $\mathrm{L}^{\mathrm{p}}$-spaces, bounded point evaluation, superharmonic function, balayage, Borel measure, Green function, Green potential, fine topology, thin, potential theoretic capacity, polar set. 1980 AMS SUBJECT CLASSIFICATION CODES. 31A05, 46E30, and 46E15.

\section{INTRODUCTION}

Subharmonic and superharmonic functions have been useful in solving the Dirichlet

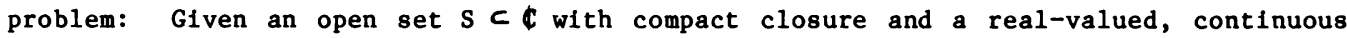
function $h$ defined on $S$, find a function $v$ harmonic in $S$ and continuous on the closure of $S$ such that

$$
v(x)=h(x) \text { for each } x \in 2 S \text {. }
$$

0. Perron showed that for many sets $S$ one can get a solution by taking the supremum of the family of subharmonic functions on $S$ whose boundary values are not greater than $h(x)$. A point $x \in \partial S$ is an irregular boundary point for $S$ if and only if there is a superharmonic function $u$ on a neighborhood $D$ of $x$ such that

$$
\begin{gathered}
u(x)<\lim _{y \rightarrow x} u(y)=+\infty . \\
y \in(D \backslash S) \backslash\{x\}
\end{gathered}
$$


We will be particularly interested in those superharmonic functions that are the Green potentials of measures supported on compact subsets of 1 Using these measures we will define a capacity that is equivalent to the Wiener capacity. Hedberg, Polking, and Fernström have shown that this capacity is helpful in identifying bounded point evaluations. For compact sets ECC we will relate the existence of a bounded point evaluation $x$ on $R^{2}(E)$ to the existence of a superharmonic function in a neighborhood of $x$. We will prove that $x$ is a bounded point evaluation on $R^{2}(E)$ if and only if there is a superharmonic function $u$ such that $u(x)<\infty$, and

$$
\begin{gathered}
u(y)>|y|^{-2}|\log | y||^{-1} \\
y \in(D \backslash E) \backslash\{x\}
\end{gathered}
$$

where $D$ is a neighborhood of $x$.

\section{SUPERHARMONIC FUNCTIONS AND BALAYAGE.}

One way to define a superharmonic function $u$ is to say that $u$ is superharmonic if and only if $-u$ is subharmonic. To be more specific let $S \subset \mathbb{C}$ be open and let $u(x)$ be $a$ function defined for $x \in S$.

DEFINITION 2.1. A function $u(x)$ is called superharmonic on $S$ if for $x \in S$

(i) $u(x)<+\infty$, and $u(x) \notin+\infty$,

(ii) $u$ is lower semi-continuous, and

(iii) $u(x)>\frac{1}{2 \pi} \int_{0}^{2 \pi} u\left(x+r e^{i \theta}\right) d \theta$ whenever the disk with radius $r>0$ and center at $x$ is contained in $S$.

EXAMPLES. If $f(z)$ is analytic in $s$ and $\lambda>0$, then $-\log |f|$ and $-|f|^{\lambda}$ are superharmonic in $S$.

Although superharmonic functions need not be continuous, one can define a new topology on in which all superharmonic functions are continuous. The smallest such topology is called the fine topology. A set ECC is thin at $x$ if $x$ is not a fine limit point of E. The following theorem is part of Brelot's contribution to potential theory. For the proof see[1, p. 210].

THEOREM 2.1. A set $E$ is thin at a limit point $x$ of $E$ if and only if there is a superharmonic function $u$ on a neighborhood $D$ of $x$ such that

$$
\begin{gathered}
u(x)<\lim u(y)=+\infty . \\
y+x \\
y \in(D \backslash E) \backslash\{x\}
\end{gathered}
$$

Later we will construct a montone increasing sequence $\left\{u_{1}\right\}$ of superharmonic functions on a set $S$ that is open in the ordinary topology. By a lemma in [1, p.68] sup $u_{i}$ is either harmonic or identically $\infty$.

IEI There is a way to associate with each non-negative superharmonic function $u$ on $S$ and each set $E \subset S$ another superharmonic function that dominates $u$ on $E$ and satisfies a special property. This function can be defined so that when $E$ is compact it equals a potential with respect to the Green function of $S$. We begin by letting $G_{S}$ be the Green function of $\mathrm{S}$. Let $\mathrm{u}$ be a non-negative superharmonic function on $\mathrm{s}$. \& will denote the class of superharmonic functions on $S$. If $u \in \&_{S}$ is non-negative and $E$ is any subset of $S$, let

$$
\phi_{E}^{u}=\left\{v \in \bigotimes_{S}: v>0 \text { on } S, v>u \text { on } E\right\} \text {. }
$$

Let $R_{E}^{u}=\inf \left\{v: v \in \phi_{E}^{u}\right\}$. 
The function $R_{E}^{u}$ satisfies (i) and (iii) of the definition of superharmonic. $R_{E}^{u}$ may not be lower semi-continuous. By defining

$$
\hat{R}_{E}^{u}(x)=\lim _{y+x} \inf R_{E}^{u}(y)
$$

we get a function that is superharmonic on $S$. $\hat{R}_{E}^{u}(x)$ is called the balayage of $u$ relative to $E$ in $S$. When $E C S$ is compact, the following fact about $\hat{R}_{E}^{u}(x)$ will be useful [1, p.135]: $\hat{K}_{E}^{u}(x)$ is a Green potential, 1.e. there is a Borel measure $\mu$ on $S$ such that

$$
R_{E}^{u}(x)=\int G_{S}(x, y) d \mu(y)
$$

3. POTENTIAL THEORETIC CAPACITY.

Let $S \subset \mathbb{C}$ be an open set having a Green function $G_{S}$. Let $E \subset \mathbb{C}$ be compact and let $u$ be the function identically 1 on $S$. Then by $[1, p .138] K_{E}^{l}(x)$ is a superharmonic function on $S$ that is the potential of a measure with support in $\partial E$.

DEFINITION 3.1. The measure $\mu_{E}$ for which $\hat{R}_{E}^{1}(x)=G_{S} \mu_{E}$ is called the capacitary distribution of $\mathrm{E}$.

DEFINITION 3.2. The capacity of $E$ (relative to the set $S$ ) is defined to be $C(E)=H_{E}(E)$ with $C(\phi)=0$.

The $C$ capacity is equal to the Wiener capacity which we will denote by $C_{2} \cdot$ For more information on why $C(E)=C_{2}(E)$ see $[1$, Lemma 7.19] and [2, Chap. II]. Also, in [3, p. 160] it is shown that if $E$ is a continuum with diameter $d$, there are positive constants $K_{1}$ and $K_{2}$ depending only on the distance from $E$ to $2 S$, such that

$$
\mathrm{K}_{1} /(\log 1 / \mathrm{d})^{1 / 2}<\mathrm{C}_{2}(\mathrm{E})<\mathrm{K}_{2} /(\log 1 / \mathrm{d})^{1 / 2} \text {. }
$$

There is $a_{2} C_{2}$ capacity series that converges at the points where the complement of a set $E C \mathbb{C}$ is thin. To state this as a theorem we will need still more notation. Let $\mathrm{j}<\mathrm{k}$ be positive integers. Define

$$
\begin{aligned}
& A(j, k)=\left\{z \in c: 2^{-k}<|z|<2^{-j}\right\} \\
& \text { and } A[j, k]=\left\{z \in c: 2^{-k}<|z|<2^{-j}\right\}
\end{aligned}
$$

Now let $A_{n}=A[n, n+1]$. The next theorem is due to wiener [2]. It is a statement about thinness at an arbitrary point $x \in \mathbb{C}$. We assume after a possible translation that $x=0$. The set $E$ need not be compact.

THEOREM 3.1. (Wiener) Let E C C. Then the complement of $E$ is thin at 0 if and only if

$$
\sum_{n=1}^{\infty} n C_{2}\left(A_{n} \backslash E\right)<\infty \text {. }
$$

Fernstrom and Polking used another $C_{2}$ series to identify bounded point evaluations [4]. Let $E \subset \mathbb{C}$ be compact and let $R_{0}(E)$ denote the algebra of rational functions with poles off $E$. Let $m$ be 2-dimensional Lebesgue measure. $R^{2}(E)$ will denote the closure of $\mathrm{R}_{0}(E)$ in the norm $\mathrm{L}^{2}(\mathrm{dm})$.

DEFINITION 3.3. A point $x \in E$ is a bounded point evaluation (BPE) on $R^{2}(E)$ if there is a constant $C$ such that

$$
|f(x)|<C\left\{\int_{E}|f|^{2} d m\right\}^{1 / 2} \text { for all } f \in R_{o}(E) \text {. }
$$

The next theorem applies to an arbitrary point $x \in \mathbb{C}$. We may assume after a possible translation that $x=0$. 
THEOREM 3.2. (Hedberg, Ferström, and Polking) The point $0 \in E$ is a BPE on $R^{2}(E)$ if and only if

$$
\sum_{n=1}^{\infty} 2^{2 n} C_{2}(A \backslash E)<\infty
$$

The existence of $a \mathrm{BPE}$ at $0 \in \mathrm{E}$ is a local property; hence it is no restriction to assume that $E \subset\{z:|z|<1 / 2\}=D$. The Green function for $D$ is

$$
G_{D}(0, z)=-\log 2|z| \text { for } z \in D \backslash\{0\} \text {. }
$$

We will need several lemmas to prove our theorem. These are modified versions of lemmas which can be found with their proofs in [1, Chap. 10].

LEMMA 3.1. There is a constant $b$ independent of $j$ such that

whenever $y \in C \backslash\left\{\zeta: 2^{-j-2}<|\zeta|<2^{-j+1}\right\}$ and $z \in\left\{\zeta: 2^{-j-1}<|\zeta|<2^{-j}\right\}$ for each positive integer $j>3$.

PROOF. We will consider two cases.

Case 1. $|y|>2^{-j+1},>3$. The absolute value of $\log 2|y-z|$ is no greater than $(j-1) \log 2$. The absolute value of $\log 2|y|$ is no less than $(j-2) \log 2$. Thus the quotient does not exceed $(j-1) /(j-2)$.

Case 2. $|y|<2^{-j-2}, j>3$. Then $\log 2|y-z|$ does not exceed $(j+1) \log 2$ in absolute value. Moreover, $\log 2|y|$ is greater in absolute value than $(j+1) \log 2$. Thus the quotient does not exceed 1 . Any $b>1$ will satisfy the statement of the lemma.

LEMMA 3.2. If $S$ is an open set having a Green function $G_{S}$, and $U$ is a nonempty open set having a compact closure $\bar{U} \subset S$, there is a measure $\mu$ on $\partial U$ such that $\mu(W)=C_{2}(U)$, and $G_{S} \mu=1$ on $U$.

PROOF. Let $\left\{U_{j}\right\}$ be an increasing sequence of open sets with compact closures $K_{j}=\bar{U}_{j} \subset U$ such that $U_{j}+U$. Each set $K_{j}$ has a capacitary distribution which we denote by $\mu_{j}$. Now $C_{2}(U)=\lim _{j \rightarrow \infty} C_{2}\left(K_{j}\right)=\lim _{j \rightarrow \infty} \mu_{j}\left(K_{j}\right)$. Since $C_{2}(\bar{U})<\infty$, the measures $\mu_{j}$ are uniformly bounded. There must be a subsequence of the sequence $\mu_{j}$ which we can assume to be the sequence itself and a measure $\mu$ such that

$$
\int_{\bar{U}} f d \mu_{j}+\int_{\bar{U}} f d \mu
$$

for every function $f$ continuous on $\bar{U}$.

We claim that $\mu$ has support in $\partial$. If not, there is a compact set $S \subset U$, $S \cap \mathbb{U}=\phi$, such that $\mu(S)>0$. To get a contradiction, take a non-negative function $f$ continuous on $\bar{U}_{1}$ equal to $1 \mathrm{cn} S$ and equal to 0 on $\partial U_{j}$ for $j$ sufficiently large.

Then $\int_{\bar{U}} f d \mu>\mu(S)>0$. Since each $\mu_{j}$ is supported in $\partial U_{j}, \int_{\bar{U}} f d \mu_{j}=0$ for sufficiently large $j$. This is contradiction.

If $x \in U$, then $x \in U_{j}$ for all $j$ sufficiently large. Using continuous functions with compact support to approximate $G_{S}$, we see that $G_{S} \mu_{j}(x) \rightarrow G_{S} \mu(x)$ as $j \rightarrow \infty$ By the definition of a capacitary distribution $G_{S} \mu_{j}(x)=1$ for sufficiently large $j$; hence $G_{S} \mu(x)=1$. The proof is complete.

DEFINITION 3.4. A set $Z \subset \mathbb{C}$ is a polar set if there is an open set $U \supset Z$ and a function $u$ superharmonic on $U$ such that $\{z: u(z)=+\infty\} \supset z$.

The next two lemmas will be useful in proving that a certain $C_{2}$ capacity series converges. 
LEMMA 3.3. Let $\checkmark$ be a measure having support $F \subset D$. If $G_{D}>\alpha$ on $F$ except possibly for a polar subset of $F$, then $V(F)>\alpha \hat{\varepsilon}_{2}(F)$.

For the proof see $[1$, p. 219].

LEMMA 3.4. If $v$ is a finite measure on $D$ such that $v=G_{D} \nu$ is finite at 0 , there is a constant a depending only on $v$, such that

$$
\begin{aligned}
& \int G_{D}(y, z) d \cup(x)<\alpha \\
& D \backslash A(j-1, j+2)
\end{aligned}
$$

for all y $\in D \cap A[j, j+1]$.

PROOF. Since $G_{D}(y, z)<-\log |y-z|$, we may prove the lemma by proving the inequality with $G_{D}$ replaced by $-\log |y-z|$. By Lemma 3.1 . there is a constant b, independent of $j>3$, such that

$$
\begin{gathered}
\int-\log |y-z| d v(y)=\int[-\log 2|y-z|+\log 2] d v(y) \\
D \backslash A(j-1, j+2) \quad D \backslash A(j-1, j+2) \\
<-b \int \log 2|y| d v(y)+(\log 2) v(D) \\
\quad D \backslash A(j-1, j+2) \\
<-b \int \log 2|y| d v(y)+(\log 2) v(D)
\end{gathered}
$$

for all $z \in D \cap A[j, j+1]$. Since we have assumed that $-\int_{D} \log 2|y| d v(y)$ is finite, we can take $\alpha=-b \int_{D} \log 2|y| d v(y)+(\log 2) v(D)$.

4. THE MAIN THEOREM.

Let $E \subset C$ be compact. The property of being a BPE on $R^{2}(E)$ is local property and is invariant under translation. In stating our theorem about an arbitrary point $x \in E$, we may therefore assume that $E \subset\{z:|z|<(d / 2)\}=D$ and that $x=0$. We will combine Theorem 3.2. with ideas of Wiener and Brelot to prove:

THEOREM 4.1. The point $0 \in E$ is a $B P E$ on $R^{2}(E)$ if and only if there is a function $u$ superharmonic in $D$ such that $u(0)<\infty$, and

$$
\begin{gathered}
u(y)>|y|^{-2}|\log | y||^{-1} . \\
y \in D \backslash E
\end{gathered}
$$

PROOF. Suppose that $0 \in E$ is a BPE on $R^{2}(E)$.

Then by Theorem 3.2 .

$$
\sum_{n=1}^{\infty} 2^{2 n} C_{2}\left(A_{n} \backslash E\right)<\infty_{.}
$$

Let $\left\{\varepsilon_{n}\right\}$ be a sequence of positive numbers such that

$$
\sum_{n=1}^{\infty} \varepsilon_{n} 2^{2 n}<\infty .
$$

For each $n>1$ let $U_{n}$ be a nonempty open subset of $D$ containing $A_{n} E$ such that $\overline{\mathrm{U}}_{\mathrm{n}} \subset \mathrm{D}$, and the following conditions hold:

$$
\begin{aligned}
& U_{n} \cap\left\{\zeta:|\zeta|<\frac{1}{2^{n+2}}\right\}=\phi \text {, and } \\
& C_{2}\left(U_{n}\right)<C_{2}\left(A_{n} \backslash E\right)+\varepsilon_{n} .
\end{aligned}
$$


Then $\quad \sum_{n=2}^{\infty} 2^{2 n} C_{2}\left(U_{n}\right)<\infty$.

We will obtain the required function $u$ as the limit of a sequence of superharmonic functions. Let $G$ denote the Green function for $D$. By Lemma 3.2. there is a measure $\mu_{n}$ with support in $\partial_{n}$ such that

We have

$$
\mu_{n}\left(U_{n}\right)=C_{2}\left(U_{n}\right) \text {, and } G_{n}=1 \text { on } U_{n} \text {. }
$$

$$
\begin{aligned}
G \mu_{n}(0)=\int_{\partial U_{n}} G(0, z) d \mu_{n}(z) & =\int_{\partial U_{n}}-\log 2|z| d \mu_{n}(z) \\
& <(n+1)(\log 2) \mu_{n}\left(\partial U_{n}\right) \\
& =(n+1)(\log 2) C_{2}\left(U_{n}\right) .
\end{aligned}
$$

For $m>1$, define $u_{m}=\sum_{n=2}^{m} 2^{2 n+2}((n+1) \log 2)^{-1} G \mu_{n}$. By a remark in Section 2

the $u_{m}$ tend to a function $u$ that is superharmonic in $D$ and satisfies $u(0)<\infty$. Since $G \mu_{n}=1$ on $U_{n} \supset A_{n} \backslash E$,

$$
u>2^{2 k+2}((k+1) \log 2)^{-1} \text { on } \underset{n \geqslant k}{U} A_{n} \backslash E \subset \notin \backslash E
$$

for each $k>2$. Thus

$$
\underset{y \in D \backslash E}{u(y)}>|y|^{-2}|\log | y||^{-1}
$$

Now suppose there is a function $u$ superharmonic in $D$ such that

$u(0)<\infty$, and

$$
y_{D \backslash E}^{u(y)}>|y|^{-2}|\log | y||^{-1}
$$

The function $u$ is lower semi-continuous on $D$; hence we may assume by taking a smaller $D$ if necessary that $u$ is positive on D. Moreover, the Riesz Decomposition Theorem implies that $u=G_{\mu}+h$ where $\mu$ is a measure supported on $D$, and $h$ is harmonic in $D$. Since $h$ is bounded, we may assume that $u=G \mu$. By Theorem 3.2. It suffices to show that

$$
\sum 2^{2 n} C_{2}\left(A_{n} \backslash E\right)<\infty
$$

For $n>2$ consider the open sets

$$
U_{n}=\left\{z: u(z)>\frac{2^{2 n}}{n \log 2}\right\} \supset A_{n} \backslash E
$$

and the sets $v_{n}=U_{n} \cap\left(A_{n} \backslash E\right)$. Since $V_{n} \supset A_{n} \backslash E$, it suffices to show that

$$
\sum_{n=2}^{\infty} 2^{2 n} C_{2}\left(v_{n}\right)<\infty
$$

Let $\mathrm{K}_{\mathrm{n}}$ be a compact subset of $\mathrm{V}_{\mathrm{n}}$ such that

$$
C_{2}\left(K_{n}\right)>C_{2}\left(V_{n}\right)-\varepsilon_{n}
$$


Then it is sufficient to prove that

$$
\sum_{n=2}^{\infty} 2^{2 n} c_{2}\left(k_{n}\right)<\infty .
$$

One way to prove that this series converges is to prove that

$$
\sum_{n=1}^{\infty} 2^{8 n+2 l} C_{2}\left(k_{4 n+\ell}\right)<\infty
$$

for $\ell=0,1,2$, and 3 . We will do this for $\ell=0$; the 3 other cases are similar.

Let $K$ be the compact set defined by $K=\underset{n>2}{U} K_{4 n} U\{0\}$. Let $w=\hat{R}_{K}^{u}$. Since $u(0)>\hat{R}_{K}^{u}(0)=w(0), w(0)<\infty$. Now $w$ is the Green potential of a measure $v$ with support in $K\left[1\right.$, p. 135]. We note that $\cup\left(D \backslash \cup \mathrm{K}_{4 n}\right)=0$ because $w(0)<\infty$. For each $\mathbf{n}>2$

$$
w=\int_{K_{4 n}} G(\cdot, z) d v(z)+\int_{\substack{m \neq n \\ K_{k m}}} G(\cdot, z) d v(z)
$$

provided we can show that the sets $k_{4 n}, n>2$, are disjoint.

Since $V_{4 n} \subset A_{4 n}$,

$$
\begin{aligned}
k_{4 n} \subset v_{4 n} & <\left\{z: 2^{-4 n-2}<|z|<2^{-4 n+1}\right\} \\
& =\left\{z: 2^{-4 n-3}<2^{-1}|z|<2^{-4 n}\right\}
\end{aligned}
$$

Suppose that $m \neq n$. Then

$$
K_{4 m} \subset\left\{z: 2^{-4 m-3}<2^{-1}|z|<2^{-4 m}\right\}
$$

If $m=n+k$ with $k>0$, then

$$
\begin{aligned}
& 2^{-4 m}=2^{-4 n-4 k}<2^{-4 n-3}, \text { and } \\
& k_{4 m} \subset D \backslash\left\{z: 2^{-4 n-3}<2^{-1}|z|<2^{-4 n}\right\} \text {. } \\
& \text { If } m=n-k \text { with } k>0 \text {, then }
\end{aligned}
$$

$$
\begin{aligned}
& 2^{-4 m-3}=2^{-4 n+4 k-3}>2^{-4 n} \text {, and } \\
& K_{4 m} C D \backslash\left\{z: 2^{-4 n-3}<2^{-1}|z|<2^{-4 n}\right\} .
\end{aligned}
$$

In either case $k_{4 m} \subset D \backslash A(4 n-1,4 n+2)$. The sets $k_{4 n}, n>2$, are disjoint.

Since $U K_{4 m} \subset D \backslash A(4 n-1,4 n+2)$, Lemma 3.4. Implies there 18 a constant $B$ depending only on $v$ such that

$$
\begin{aligned}
& \text { for all } y \in D \cap A_{4 n} . \quad \\
& \text { Thus } \\
& \quad \int_{m \neq n} G(y, z) d v(z)<B \\
& w(y)<B+\int_{K_{4 n}} G(y, z) d v(z)
\end{aligned}
$$

for all $y \in D \cap A_{4 n}$. The functions $w$ and $u$ are equal on $K$ except perhaps for a polar set $\mathrm{Z} \subset \mathrm{K}$. Thus 


$$
\underset{y \in K \backslash Z}{w(y)}>|y|^{-2}|\log | y||^{-1}
$$

Choose an integer $N_{0}$ such that

$$
\frac{2^{8 n}}{4 n \log 2}>B \text { for } n>N_{0}
$$

Then $\int_{K_{4 n}} G(y, z) d V(z)>\frac{2^{8 n}}{4 n \log 2}-\beta>0$ for all $y \in K_{4 n} \backslash Z$, and $n>N_{0}$. By

LEMMA 3.3 .

$$
v\left(K_{4 n}\right)>\left(\frac{2^{8 n}}{4 n \log 2}-\beta\right) c_{2}\left(K_{4 n}\right) \text { for all } n>N_{0}
$$

Hence

$$
\sum_{n \geqslant 0} n v\left(K_{4 n}\right)>\sum_{n \geqslant 0}\left(\frac{2^{8 n}}{4 n \log 2}-\beta\right) n c_{2}\left(K_{4 n}\right) .
$$

The series $\left[\mathrm{nC}_{2}\left(\mathrm{~K}_{4 \mathrm{n}}\right)\right.$ converges because the hypothesis on $\mathrm{u}$ implies that the complement of $\mathrm{E}$ is thin at 0 , and Theorem 3.1. applies. It remains only to show that the series

$$
\sum n \cup\left(K_{4 n}\right) \text { converges. }
$$

Now

$$
\begin{gathered}
-\int \log 2|z| d v(z)>\sum_{n=2}^{\infty} \int_{4 n}-\log 2|z| d v(z) \\
>\sum_{n=2}^{\infty}(4 n-1)(\log 2) v\left(k_{4 n}\right) .
\end{gathered}
$$

Note that

$$
\infty>w(0)=\int G(0, z) d v(z)=-\int \log 2|z| d v(z) \text {. }
$$
Thus the series $\sum_{n=2}^{\infty}(4 n-1) \log 2 v\left(k_{4 n}\right)$ converges, and so does the series $\sum_{n=1}^{\infty} n \cup\left(K_{4 n}\right)$.
This completes the proof.

\section{REFERENCES}

1. HELMS, L.L., Introduction to Potential Theory, Robert E. Krieger Publishing Company (1975).

2. LANDKOF, N.S., Foundations of modern Potentlal Theory, translated from the Russian by A.P. Doohovskoy, Springer-Verlag (1972).

3. HEDBERG, L.I., Approximation in the mean by analytic functions, Trans. Amer. Math. Soc., 163, (1972), 157-171.

4. FERNSTROM, C. and POLKING, J.C., Bounded point evaluations and approximation in $\mathrm{L}^{\mathrm{P}}$ by solutions of elliptic partial differential equations, J. Functional analysis, $28,(1978), 1-20$. 


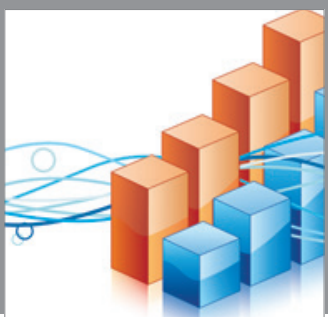

Advances in

Operations Research

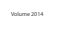

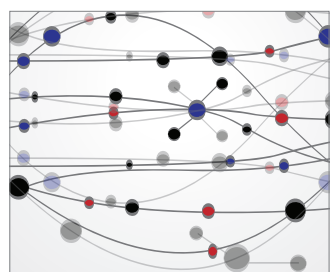

\section{The Scientific} World Journal
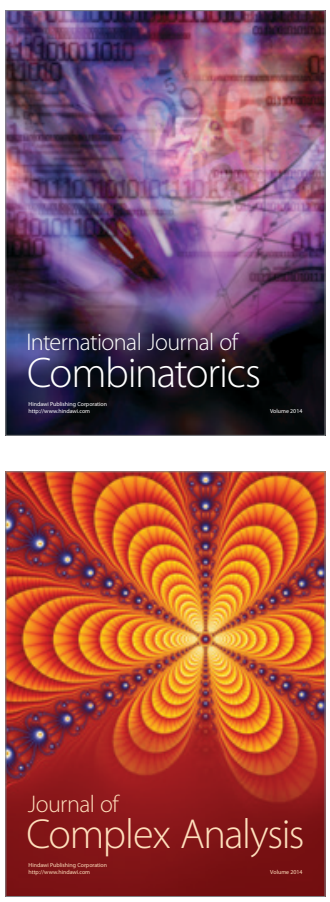

International Journal of

Mathematics and

Mathematical

Sciences
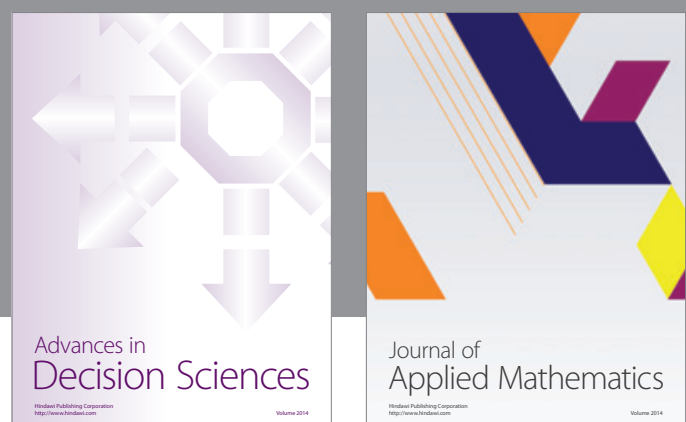

Journal of

Applied Mathematics
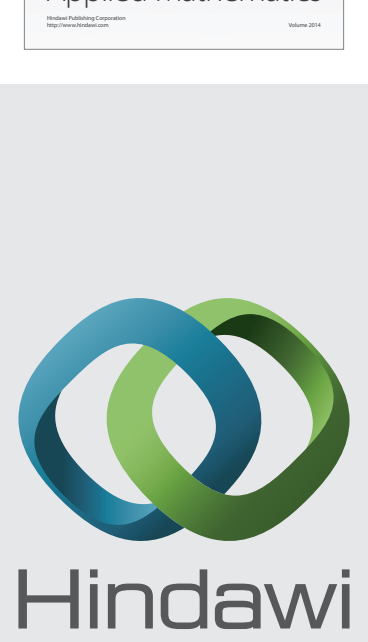

Submit your manuscripts at http://www.hindawi.com
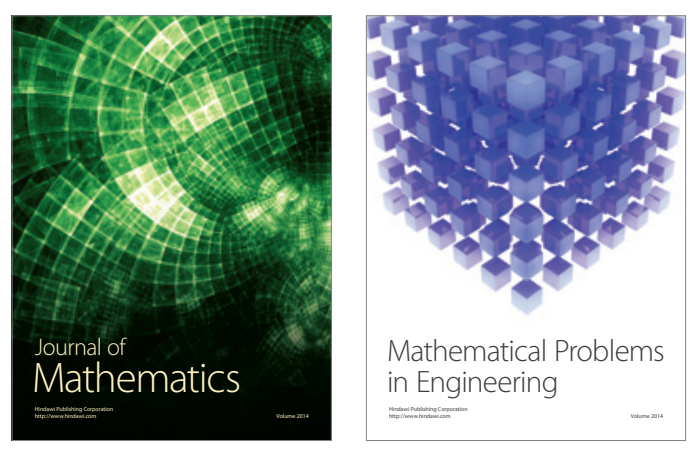

Mathematical Problems in Engineering
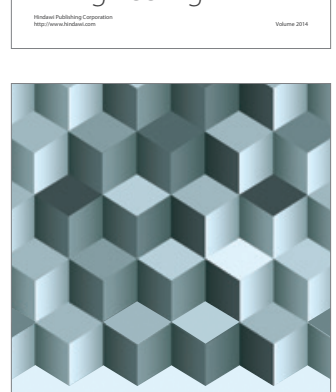

Journal of

Function Spaces
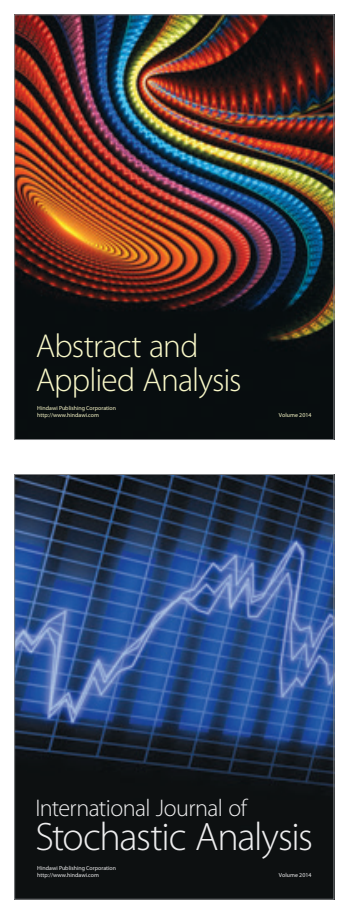

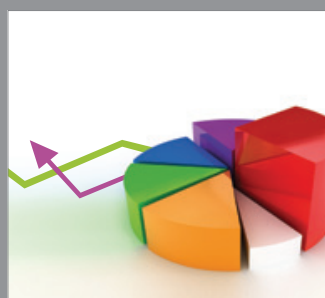

ournal of

Probability and Statistics

Promensencen
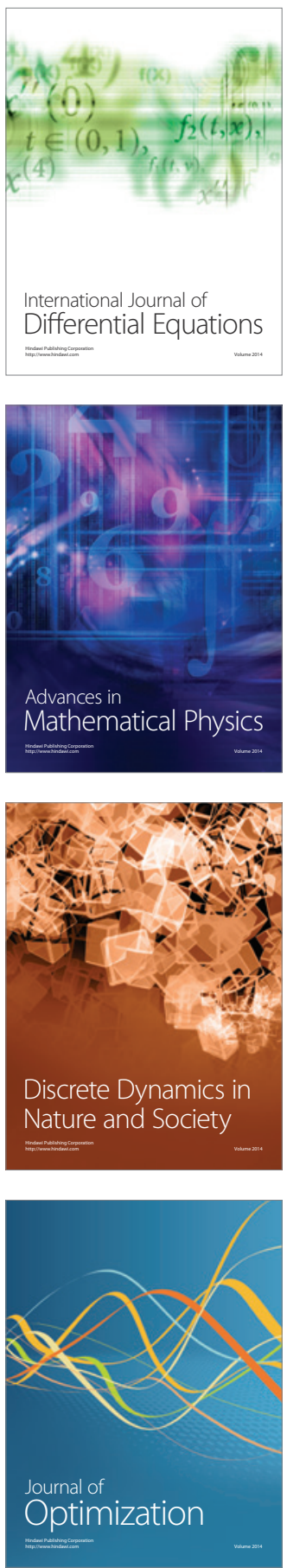